\title{
Optimized Detection of High-Dimensional Entanglement
}

\author{
Xiao-Min $\mathrm{Hu}$ (胡晓敏), ${ }^{1,2,{ }^{*}}$ Wen-Bo Xing (邢文博), ${ }^{1,2,{ }^{*}}$ Yu Guo (郭钰), ${ }^{1,2}$ \\ Mirjam Weilenmann $\odot,{ }^{3}$ Edgar A. Aguilar, ${ }^{3}$ Xiaoqin Gao (高小钦) $\odot,{ }^{3,4,5, \dagger}$ Bi-Heng Liu (柳必恒), ${ }^{1,2, *}$ \\ Yun-Feng Huang (黄运锋), ${ }^{1,2}$ Chuan-Feng Li (李传锋), ${ }^{1,2,}$ Guang-Can Guo (郭光灿), ${ }^{1,2}$ \\ Zizhu Wang (王子竹) ${ }^{6,\|,\|}$ and Miguel Navascués $\oplus^{3, \pi}$ \\ ${ }^{1}$ CAS Key Laboratory of Quantum Information, University of Science and Technology of China, Hefei 230026, China \\ ${ }^{2}$ CAS Center For Excellence in Quantum Information and Quantum Physics, University of Science and Technology of China, \\ Hefei 230026, People's Republic of China \\ ${ }^{3}$ Institute for Quantum Optics and Quantum Information-IQOQI Vienna, Austrian Academy of Sciences, \\ Boltzmanngasse 3, 1090 Vienna, Austria \\ ${ }^{4}$ Vienna Center for Quantum Science and Technology (VCQ), Faculty of Physics, University of Vienna, \\ Boltzmanngasse 5, 1090 Vienna, Austria \\ ${ }^{5}$ Department of Physics, University of Ottawa, Advanced Research Complex, \\ 25 Templeton Street, K1N 6N5 Ottawa, Ontario, Canada \\ ${ }^{6}$ Institute of Fundamental and Frontier Sciences, University of Electronic Science and Technology of China, \\ Chengdu 610054, China
}

(Received 27 November 2020; revised 26 September 2021; accepted 26 October 2021; published 22 November 2021)

\begin{abstract}
Entanglement detection is one of the most conventional tasks in quantum information processing. While most experimental demonstrations of high-dimensional entanglement rely on fidelity-based witnesses, these are powerless to detect entanglement within a large class of entangled quantum states, the so-called unfaithful states. In this Letter, we introduce a highly flexible automated method to construct optimal tests for entanglement detection given a bipartite target state of arbitrary dimension, faithful or unfaithful, and a set of local measurement operators. By restricting the number or complexity of the considered measurement settings, our method outputs the most convenient protocol which can be implemented using a wide range of experimental techniques such as photons, superconducting qudits, cold atoms, or trapped ions. With an experimental quantum optics setup that can prepare and measure arbitrary highdimensional mixed states, we implement some three-setting protocols generated by our method. These protocols allow us to experimentally certify two- and three-unfaithful entanglement in four-dimensional photonic states, some of which contain well above $50 \%$ of noise.
\end{abstract}

DOI: 10.1103/PhysRevLett.127.220501

Entanglement is the bedrock of most quantum information processing protocols $[1,2]$. It is a key resource in quantum teleportation [3], entanglement-based quantum key distribution (QKD) [4] and quantum communication complexity [5]. Generating high-quality entangled states and detecting them reliably is a crucial prerequisite to conduct any quantum communication task. However, entanglement detection is a computationally hard problem for high-dimensional systems [6,7]. There are only a few known general methods, all of which come with high computational costs [8-11]. Most experimental entanglement detection protocols, especially those which aim to detect high-dimensional entanglement, use linear witnesses

Published by the American Physical Society under the terms of the Creative Commons Attribution 4.0 International license. Further distribution of this work must maintain attribution to the author(s) and the published article's title, journal citation, and DOI. based on the fidelity between the generated state and a (pure) target state, see, e.g., Refs. [12-15]. Fidelity-based witnesses have been shown to work well for the states generated in current experiments, with only a constant number of measurement settings needed to measure each witness [16]. However, the recent discovery of unfaithful entanglement [17], which cannot be detected by fidelitybased witnesses, has changed the status quo.

Unfaithful entanglement is not the exception, but the norm: almost all high-dimensional bipartite entangled states are unfaithful [17]. This situation posed a conundrum for theorists and experimentalists alike: how to supplement fidelity-based witnesses with experiment-friendly protocols that are capable of detecting unfaithful entanglement? Here we tackle this problem by designing a method to automatically search for optimal protocols for certifying high-dimensional bipartite entanglement, including the unfaithful kind, with only a few local measurements. Specifically, using any bipartite target state $\rho_{\mathrm{AB}}$, a set of 
local measurement operators $\left\{N^{x}\right\}_{x}$, and the entanglement dimension $D+1$ of $\rho_{\mathrm{AB}}$ to be certified as input, our method constructs a two-element positive operator-valued measure (POVM), $M=\left(M_{\mathrm{C}}, M_{\mathrm{U}}\right)$, where outcome $\mathrm{C}$ stands for certified $D+1$-dimensional entanglement and $\mathrm{U}$ for "uncertified." This POVM can be conducted through a one-way local operations and classical communication (LOCC) protocol, and the probability of mistakenly reporting outcome $C$ when a state of Schmidt rank $D$ or lower was prepared or outcome $\mathrm{U}$ when the target state $\rho_{\mathrm{AB}}$ was prepared is minimal as guaranteed by convex optimization theory. If we conduct several experimental implementations of the protocol, then the number of occurrences of outcome $\mathrm{C}$ can be used to certify, with high statistical confidence, that the generated state $\rho_{\exp }$ has Schmidt rank at least $D+1$, even if the protocol's measurement settings are far from tomographically complete. These protocols can be implemented in a wide variety of physical systems, especially where the presence of noise in high-dimensional entangled states renders them unfaithful thus making their certification with fidelity-based witnesses impossible.

We experimentally tested our method with a dozen fourdimensional bipartite target states, each belonging to one of two groups. The archetype of the first group has threedimensional entanglement (three-entangled) but is threeunfaithful and the second group is two entangled but two unfaithful. Using our method, the entanglement dimension of the first group can be certified with 3 commonly used measurement settings per side and estimating 22 to 44 different probabilities. For the two unfaithful states, a different set of 3 measurement settings per side and 89 probabilities are needed.

Method for generating optimal protocols for highdimensional entanglement detection.-We start from the following premise which covers the most basic experimental scenario: two parties, Alice and Bob, want to certify the entanglement dimension of a shared quantum state $\rho_{\mathrm{AB}}$ with local dimension $d$. Each of them can perform $m$ local measurements in their part of the lab, with each measurement producing one of $d$ possible outcomes. We can hence identify each measurement $x \in\{1, \ldots, m\}$ by the set of POVM elements $N^{x} \equiv\left\{N_{a \mid x}: a \in\{1, \ldots, d\}\right\}$. We further allow Alice and Bob to carry out 1-way LOCC protocols. Namely, we allow Alice to measure first and then communicate her measurement setting $x$ and outcome $a$ to Bob. With this information, Bob decides which measurement $y$ to conduct in his lab, call $b$ his measurement result. Alice and Bob's guess on the entanglement of their shared state will be a nondeterministic function of $a, b, x, y$.

More formally, call $S_{D}$ the set of all quantum states $\sigma_{A B}$ that admit a decomposition of the form

$$
\sigma_{\mathrm{AB}}=\sum_{i} \lambda_{i}\left|\psi_{i}\right\rangle\left\langle\left.\psi_{i}\right|_{A B},\right.
$$

where each state $\left|\psi_{i}\right\rangle$ has Schmidt rank $D$ or lower, and the weights $\left\{\lambda_{i}\right\}$ satisfy $\lambda_{i} \geq 0$, for all $i$ and $\sum_{i} \lambda_{i}=1$. Any state that does not belong to $S_{D}$ has entanglement dimension at least $D+1$.

Let $\rho_{\mathrm{AB}}$ be such a state: $\rho_{\mathrm{AB}} \notin S_{D}$, and let $p_{\text {I }}, p_{\text {II }} \in[0,1]$. We first consider one-shot one-way LOCC measurement protocols for Alice and Bob, with possible outcomes $C$ (certified) and $U$ (uncertified), such that (1) If the state $\sigma$ shared by Alice and Bob belongs to $S_{D}$, the probability that they output $\mathrm{C}$ is bounded by $p_{\mathrm{I}}$. (2) If the state shared by Alice and Bob is indeed the target state $\rho_{\mathrm{AB}}$, the probability that Alice and Bob output $\mathrm{U}$ is bounded by $p_{\text {II }}$.

The conditions above constitute a hypothesis test, with $p_{\text {I }}$ and $p_{\text {II }}$ playing the roles of type-I (false positive) and type-II (false negative) errors. Using the newly developed quantum preparation games [18], we can recast our search for one-shot one-way LOCC protocols that minimize the sum $p_{\text {I }}+p_{\text {II }}$ into the following optimization problem (see Ref. [19] for more theoretical analysis):

$$
\begin{aligned}
\text { Minimize } & p_{\mathrm{I}}+p_{\mathrm{II}} \\
\text { subject to } & \operatorname{tr}\left(M_{\mathrm{U}} \rho_{\mathrm{AB}}\right)=p_{\mathrm{II}}, \\
& \Pi_{D}\left(p_{\mathrm{I}} \mathbb{I}_{A B}-M_{\mathrm{C}}\right) \Pi_{D}^{\dagger} \\
& =\Lambda_{A A^{\prime} B^{\prime} B}^{1}+\left(\Lambda_{A A^{\prime} B^{\prime} B}^{2}\right)^{T_{B B^{\prime}}}+\lambda\left(\Pi_{D} \Pi_{D}^{\dagger}-\frac{\mathbb{I}}{D}\right), \\
& M_{\mathrm{C}}=\sum_{x, y, a, b} P(x, y, c=\mathrm{C} \mid a, b) N_{a \mid x} \otimes N_{b \mid y}, \\
& M_{\mathrm{U}}=\mathbb{I}_{A B}-M_{\mathrm{C}}, \\
& \Lambda^{1}, \Lambda^{2} \succeq 0, P(x, y, c \mid a, b) \geq 0, \\
& \sum_{c} P(x, y, c \mid a, b)=P(x, y \mid a), \\
& \sum_{y} P(x, y \mid a)=P(x), \quad \sum_{x} P(x)=1 .
\end{aligned}
$$

Here auxiliary systems $A^{\prime}, B^{\prime}$ are assumed to have dimension $D$, and $\Pi_{D}=\mathbb{I}_{A} \otimes\left|\psi_{D}^{+}\right\rangle_{A^{\prime} B^{\prime}} \otimes \mathbb{I}_{B}$, with $\left|\psi_{D}^{+}\right\rangle=$ $\sum_{i=1}^{D}|i\rangle|i\rangle$ being the non-normalized maximally entangled state in $\mathbb{C}^{D} \times \mathbb{C}^{D} . T_{B B^{\prime}}$ denotes the partial transpose over systems $B B^{\prime}$. The optimization variables $P(x, y, c \mid a, b)$ represent a collection of probability distributions, with $x, y \in\{1, \ldots, m\}, a, b \in\{1, \ldots, d\}, c \in\{\mathrm{C}, \mathrm{U}\}$. The meaning of $\Lambda^{1}, \Lambda^{2}$ and a more complete derivation can be found in the Supplemental Material [19].

In effect, Eq. (2) describes the following problem: given the state $\rho_{\mathrm{AB}}$ shared by Alice and Bob, together with their allowed local measurements $N_{a \mid x}, N_{b \mid y}$, what is the best one-way LOCC strategy that maximizes the chance they can correctly certify the entanglement dimension of $\rho_{\mathrm{AB}}$ to be $D+1$ while at the same time minimizes the chance they make mistakes? The solution to this problem is a feasible 


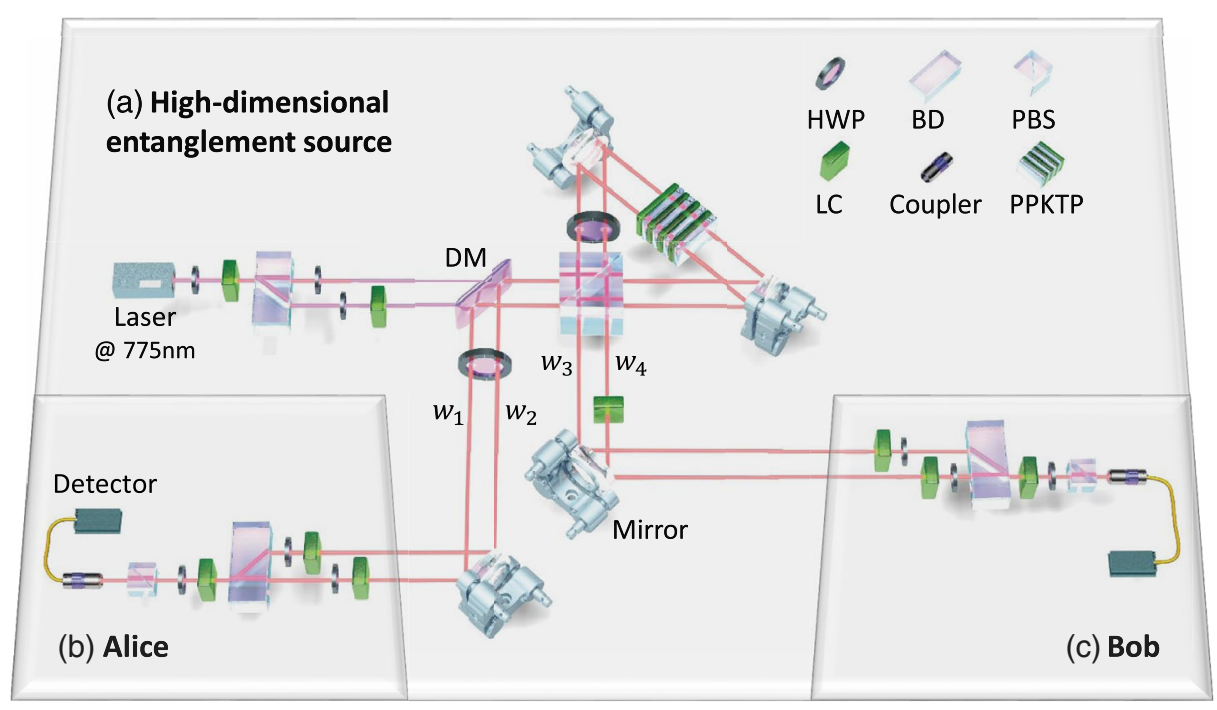

FIG. 1. Experimental setup for generating the unfaithful states. (a) Preparation of a four-dimensional entanglement source @ 1550 nm. After two lenses (not shown), the continuous-wave (cw) laser beam (@ 775 nm with power @ 200 mW) is focused into a small beam with waist radius of $\approx 0.7 \mathrm{~mm}$. With a beam-displacer (BD), the beam is separated into two paths. Then, these two beams are injected into a Sagnac interferometer, which generates a two-photon polarization-based spontaneous parametric down-conversion (SPDC) entangled state $(1 / \sqrt{2})(|H V\rangle+|V H\rangle)$ in each path [24] by a type-II PPKTP crystal [1 $\mathrm{mm} \times 7 \mathrm{~mm} \times 10 \mathrm{~mm}$, the polling period is $46.2 \mu \mathrm{m}$, and the temperature is set at $35^{\circ} \mathrm{C}$. In our experiment, the full width at half maximum of the down-converted photons is $\approx 2 \mathrm{~nm}$.]. After a half-wave plate (HWP) @ $45^{\circ}$, each entangled state is rotated to $(1 / \sqrt{2})(|H H\rangle+|V V\rangle)$, which is encoded into the paths $w_{1}\left(w_{3}\right)$ and $w_{2}\left(w_{4}\right)$, respectively. If we define the path $w_{1}\left(w_{3}\right)$ with $H$-polarized photon as $|0\rangle, w_{1}\left(w_{3}\right)$ with $V$-polarized photon as $|1\rangle, w_{2}\left(w_{4}\right)$ with $H$-polarized photon as $|2\rangle$, and $w_{2}\left(w_{4}\right)$ with $V$-polarized photon as $|3\rangle$, then a four-dimensional maximally entangled state is generated. Afterwards, to prepare the state $\rho_{\mathrm{UNF}}^{p}$, we need to change the angles of first three HWPs and liquid crystals (LCs) together with their voltages. If we only consider the paths $w_{1}$ and $w_{3}$, then we obtain the state $\left|\Psi_{2}\right\rangle$. An additional light source is introduced as white noise mixed with the pure state $\left|\Psi_{2}\right\rangle$ to generate the states $\rho_{\mathrm{ISO} 2}^{p}$. (b) and (c) Setups for Alice's and Bob's local measurements. Each party has three LCs, three HWPs, a BD, a PBS and a single-photon superconducting detector. By adjusting the voltages of the LCs and the angles of the HWPs, different measurement bases can be implemented.

sum of $p_{\text {I }}$ and $p_{\text {II }}$, with the operational description of the protocol achieving this sum encoded in the minimizer $P^{\star}(x, y, c \mid a, b)$. The optimization algorithm also returns the optimal $p_{\text {I }}$ satisfying the first condition of the hypothesis test. This allows us to compute the $p$ value of the null hypothesis after $n$ experimental repetitions of the protocol. To solve Eq. (2), which is a semidefinite program (SDP), any number of readily available solvers such as MOSEK [23] can be used, which not only output the numerical solution of the problem, but also rigorous upper and lower bounds on its optimal value. The only practical limitation is the physical dimension $d$ and the entanglement dimension $D$, even though the method itself is valid for any $d$ and $D$. The protocols used in our experiment were computed on a normal desktop computer in less than $30 \mathrm{sec}$. In fact, problem (2) is just the first level in a hierarchy of SDPs that output feasible one-way LOCC protocols with provable type-I error $p_{\text {I }}$ and decreasing sum $p_{\text {I }}+p_{\text {II }}$. In the infinite limit, the hierarchy returns the measurement protocol that minimizes $p_{\text {I }}+p_{\text {II }}$. For small dimensions, the first level already provides good enough protocols for $D+1$-dimensional entanglement detection. Additional information such as the complete description of the hierarchy, the meaning of $\Lambda^{1}, \Lambda^{2}$ in Eq. (2), and a benchmark of the method with random target states and different sets of measurement settings can be found in Ref. [19].

Since our experimental setup does not allow us to switch the measurement settings dynamically, the LOCC protocols are not implemented directly. Instead, under the assumption that the source produces the same target state $\rho^{\exp }$ in every experimental round, we estimate the operator averages $\left\langle N_{a \mid x} \otimes N_{b \mid y}\right\rangle_{\text {exp }}$, for $a, b, x, y$. With these averages we compute the probability $p_{\text {II }}^{\exp }$ of obtaining result $\mathrm{U}$, had we implemented the optimal one-way LOCC protocol $P^{\star}(x, y, c \mid a, b)$ once. If $p_{\text {II }}^{\exp }$ is such that $p_{\text {I }}+p_{\text {II }}^{\exp }<1$, then we can conclude that $\rho^{\exp }$ has entanglement dimension at least $D+1$.

Experimental certification of unfaithful entanglement.The 2 types of target states we selected for experimental testing are

$\rho_{\mathrm{UNF}}^{p}=(1-p)\left|\Psi_{3}\right\rangle\left\langle\Psi_{3}\right|+p\left(\frac{|23\rangle+|32\rangle}{\sqrt{2}}\right)\left(\frac{\langle 23|+\langle 32|}{\sqrt{2}}\right)$, 


$$
\rho_{\mathrm{ISO} 2}^{p}=(1-p)\left|\Psi_{2}\right\rangle\left\langle\Psi_{3}\right|+\frac{p}{16} \mathbb{I}_{4} \otimes \mathbb{I}_{4},
$$

with $\left|\Psi_{d}\right\rangle$ denoting the $d$-dimensional maximally entangled state $\left|\Psi_{d}\right\rangle=(1 / \sqrt{d}) \sum_{i=0}^{d-1}|i i\rangle$. The state $\rho_{\mathrm{UNF}}^{p}$ is a mixture of pure states. Experimental methods which allow preparing this kind of high fidelity mixed states would be particularly useful because currently there are very few feasible options. We here present a general method that mixes arbitrary bipartite pure states by rapidly switching the electro-optical modulation elements (See the Supplemental Material [19] for more information).

Using the computational tests SDP1 and SDP2 in Ref. [17], we can certify that $\rho_{\mathrm{UNF}}^{0.2}, \rho_{\mathrm{UNF}}^{0.4}, \rho_{\mathrm{UNF}}^{0.6}$ is three entangled but three unfaithful while the states $\rho_{\mathrm{ISO} 2}^{0.6}, \rho_{\mathrm{ISO} 2}^{0.7}, \rho_{\mathrm{ISO} 2}^{0.8}$ are two entangled but two unfaithful. The states $\rho_{\text {ISO2 }}^{p}$ model the situation where high-dimensional noise creeps into the preparation of entangled qubits, making them unfaithful thus unsuitable for fidelity-based witnesses. For such states, our method shows that entanglement detection is possible with as much as over $80 \%$ of noise, which is confirmed by our experiment as shown in Fig. 2.

The target states are generated by the setup depicted in Fig. 1. For the state $\rho_{\mathrm{UNF}}^{p}$, we first generate the two pure states $\left|\Psi_{3}\right\rangle$ and $(1 / \sqrt{2})(|23\rangle+|32\rangle)$ using polarizationbased path degree of freedom of photons, as shown in Fig. 1(a), then mix them by rapidly switching the voltages of liquid crystals (LCs) (more information can be found in Ref. [19]). LCs play an important role in our mixed state preparation. The relative phase between the $H$ - and $V$ polarized photons can be changed by adjusting the voltage applied to the LC, which makes it behave like an HWP. The relative phase between $H$ and $V$ varies from 0 , when the applied voltage is $V_{I}$, to $\pi$, for $V_{\pi}$. The polarization is stabilized by keeping $V_{I}$ stable for the three LCs.

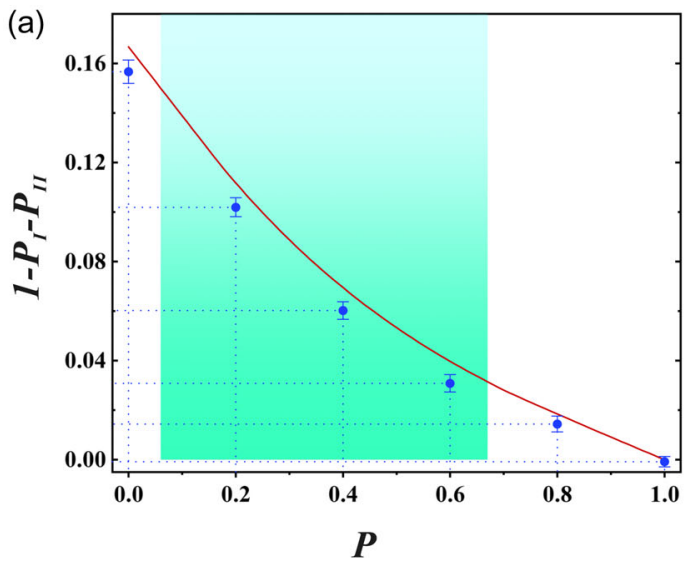

By controlling the loading time of different phases, we can prepare $\rho_{\mathrm{UNF}}^{p}$ with different values of $p$ (more information can be found in Ref. [19]).

To generate the states $\rho_{\mathrm{ISO} 2}^{p}$, we mix a two-dimensional maximally entangled state $\left|\Psi_{2}\right\rangle$ generated by the pump with four-dimensional white noise emitted by independent sources [13,25] (more information can be found in Ref. [19]).

After the states are generated, we performed tomographic reconstructions of their density matrices using maximum-likelihood estimation (MLE) [26,27]. The reconstructed density matrices, which can be found in Supplemental Material [19], are used to certify that the generated states are indeed unfaithful.

Only 3 measurement settings are needed in our experiment to certify the entanglement dimensions of $\rho_{\mathrm{UNF}}^{p}$ and $\rho_{\text {ISO2 }}^{p}$. The optimal 1-shot 1-way LOCC protocol using these measurement settings found by the SDP solver, in the form of a set of probability distributions $P(x, y, c \mid a, b)$, allow us to compute $p_{\mathrm{II}}^{\exp }$ for each of the states. In these optimal protocols, $P(x, y, c \mid a, b)=0$ for many combinations of $x, y, c, a, b$, meaning we only need to estimate fewer probabilities in the experiment. In general, we need far fewer probability combinations to certify the entanglement dimension of a target state than necessary using state tomography, as can be seen in Table I. For example, there are only eight probability combinations needed to certify the entanglement dimension of the four-dimensional maximally entangled state $\left|\Psi_{4}\right\rangle$. This is a big improvement over both the 256 probability combinations necessary for its tomographic reconstruction and the $2 d^{2}=32$ combinations necessary for fidelity-based entanglement dimension certification [12].

The theoretical and experimental values of $1-p_{\text {I }}-p_{\text {II }}$ are presented in Table I. If $1-p_{\text {I }}-p_{\text {II }}>0$, the state is

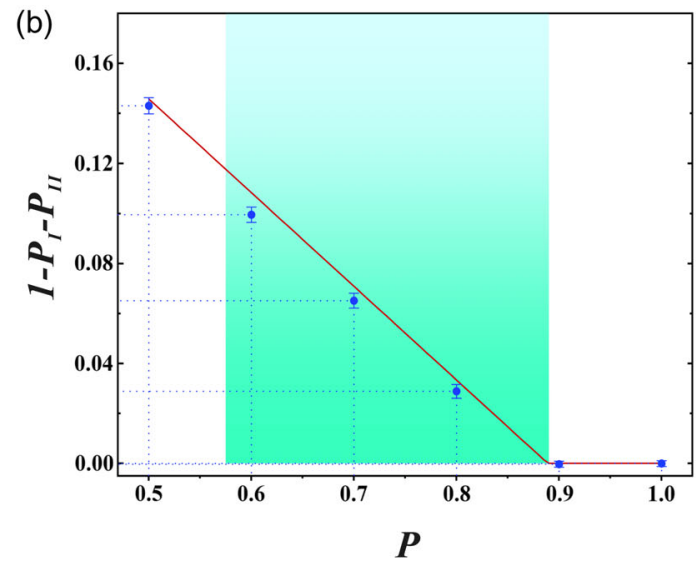

FIG. 2. Experimental results of the optimal $1-p_{\text {I }}-p_{\text {II }}$ for states. Experimental values for $p=0,0.2,0.4,0.6,0.8$, and 1 of state $\rho_{\mathrm{UNF}}^{p}$ in (a) and $p=0.5,0.6,0.7,0.8,0.9$, and 1 of state $\rho_{\mathrm{ISO}}^{p}$ in (b) are shown in blue. The solid red lines represent optimal values computed by the SDP solver. States in the green regions are three unfaithful in (a) and two unfaithful in (b). The entanglement dimension is certified when $1-p_{I}-p_{\text {II }}>0$. 
TABLE I. Experimental results. $\mathcal{N}_{\mathcal{S}}$ and $\mathcal{N}_{\mathcal{P}}$ are the number of settings and probabilities, respectively. $D$ is the entanglement dimension.

\begin{tabular}{lccccc}
\hline \hline Target state & $1-p_{\text {I }}-p_{\text {II }}$ & $1-p_{\text {I }}-p_{\text {II }}^{\exp }$ & $\mathcal{N}_{\mathcal{S}}$ & $\mathcal{N}_{\mathcal{P}}$ & $D$ \\
\hline$\rho_{\mathrm{UNF}}^{0}$ & 0.167 & $0.160 \pm 0.005$ & 3 & 38 & 3 \\
$\rho_{\mathrm{UNF}}^{0.2}$ & 0.112 & $0.102 \pm 0.004$ & 3 & 22 & 3 \\
$\rho_{\mathrm{UNF}}^{0.4}$ & 0.070 & $0.069 \pm 0.004$ & 3 & 26 & 3 \\
$\rho_{\mathrm{UNF}}^{0.6}$ & 0.040 & $0.031 \pm 0.003$ & 3 & 26 & 3 \\
$\rho_{\mathrm{UNF}}^{0.8}$ & 0.018 & $0.014 \pm 0.003$ & 3 & 44 & 3 \\
$\rho_{\mathrm{UNF}}^{1.0}$ & 0 & $-0.000 \pm 0.002$ & 3 & 144 & N/A \\
$\rho_{\mathrm{ISO} 2}^{0.5}$ & 0.146 & $0.143 \pm 0.003$ & 3 & 89 & 2 \\
$\rho_{\mathrm{ISO} 2}^{0.6}$ & 0.108 & $0.100 \pm 0.003$ & 3 & 89 & 2 \\
$\rho_{\mathrm{ISO} 2}^{0.7}$ & 0.071 & $0.065 \pm 0.003$ & 3 & 89 & 2 \\
$\rho_{\mathrm{ISO} 2}^{0.8}$ & 0.033 & $0.029 \pm 0.003$ & 3 & 89 & 2 \\
$\rho_{\mathrm{ISO} 2}^{0.9}$ & 0 & $0.001 \pm 0.001$ & 3 & 144 & N/A \\
$\rho_{\mathrm{ISO} 2}^{1.0}$ & 0 & $-0.000 \pm 0.001$ & 3 & 144 & N/A \\
$\Phi_{4}$ & 0.084 & $0.073 \pm 0.005$ & 2 & 8 & 4 \\
\hline \hline
\end{tabular}

certified to possess $(D+1)$-dimensional entanglement. However, we cannot get an valid conclusion (not applicable), if $1-p_{\text {I }}-p_{\text {II }}=0$. The slight difference between the experimental values and the theoretical curves may come from the differences of the noise profile: the theoretical curves are obtained by assuming the noise to be perfectly depolarizing, while the noise is added through an uncorrelated external light source in the experiment and it might have a less-than-ideal noise profile. The counting rate of the entanglement source is about $1250 / s$ and all data points are collected for $20 \mathrm{~s}$. The statistical errors are estimated using the Monte Carlo method.

As one can appreciate, we can certify the entanglement dimension for each target state from the observation that $p_{\text {I }}+p_{\text {II }}^{\exp }<1$ for all of them. The measurement bases and distributions $P(x, y, c \mid a, b)$ used in the experiment can be found in Ref. [19].

Conclusions.-Qubits and qudits are typically made by ignoring unused degrees of freedom in physical systems. When noise inevitably affects them, unfaithful entanglement may become unavoidable in experiments. This signifies the urgent need to identify experiment-friendly entanglement detection protocols which can also certify unfaithful entanglement. In the short time since the discovery of unfaithful entanglement, advances have already been made to study its structure and detection [28,29]. Compared to these results, our method does not specifically target unfaithful states and it is conceived to be experiment friendly. We recast the entanglement detection problem into a quantum preparation game [18] and extract from the solution of the resulting optimization problem protocols capable of certifying the entanglement dimension of bipartite high-dimensional states. By incorporating an experimental technique which can produce arbitrary high-dimensional mixed states with high fidelity, we test our protocols for four-dimensional unfaithful states. Despite having as much as $80 \%$ of noise in one state, we are able to obtain close agreements between experimental and theoretical values. Our work lays the foundation for the efficient generation, detection, and application $[30,31]$ of high-dimensional entangled states.

This work was supported by the National Key R\&D Program of China (No. 2018YFA0306703, No. 2021YFE0113100, No. 2017YFA0304100), National Natural Science Foundation of China (No. 11774335, No. 11734015, No. 11874345, No. 11821404, No. 11904357, No. 12174367), the Key Research Program of Frontier Sciences, CAS (No. QYZDY-SSW-SLH003), Science Foundation of the CAS (ZDRW-XH-2019-1), the Fundamental Research Funds for the Central Universities, USTC Tang Scholarship, Science and Technological Fund of Anhui Province for Outstanding Youth (2008085J02). M. N., M. W., and E. A. A. were supported by the Austrian Science Fund (FWF) stand-alone project P 30947. X. G. acknowledges the support of Austrian Academy of Sciences (ÖAW) and Joint Center for Extreme Photonics (JCEP).

\footnotetext{
*These two authors contributed equally to this work †xgao5@uottawa.ca "bhliu@ustc.edu.cn §cfli@ustc.edu.cn "zizhu@uestc.edu.cn "miguel.navascues@oeaw.ac.at
}

[1] R. Horodecki, P. Horodecki, M. Horodecki, and K. Horodecki, Quantum entanglement, Rev. Mod. Phys. 81, 865 (2009).

[2] O. Gühne and G. Tóth, Entanglement detection, Phys. Rep. 474, 1 (2009).

[3] C. H. Bennett, G. Brassard, C. Crépeau, R. Jozsa, A. Peres, and W. K. Wootters, Teleporting an Unknown Quantum State via Dual Classical and Einstein-Podolsky-Rosen Channels, Phys. Rev. Lett. 70, 1895 (1993).

[4] J. Yin et al., Satellite-to-Ground Entanglement-Based Quantum Key Distribution, Phys. Rev. Lett. 119, 200501 (2017).

[5] H. Buhrman, R. Cleve, S. Massar, and R. de Wolf, Nonlocality and communication complexity, Rev. Mod. Phys. 82, 665 (2010).

[6] S. Gharibian, Strong NP-Hardness of the Quantum Separability Problem, Quantum Inf. Comput. 10, 343 (2010).

[7] L. Gurvits, Classical deterministic complexity of Edmonds' problem and quantum entanglement, in Proceedings of the Thirty-Fifth Annual ACM Symposium on Theory of Computing, STOC '03 (Association for Computing Machinery, New York, New York, USA, 2003), pp. 10-19. 
[8] A. C. Doherty, P. A. Parrilo, and F. M. Spedalieri, Distinguishing Separable and Entangled States, Phys. Rev. Lett. 88, 187904 (2002).

[9] A. C. Doherty, P. A. Parrilo, and F. M. Spedalieri, Complete family of separability criteria, Phys. Rev. A 69, 022308 (2004).

[10] A. C. Doherty, P. A. Parrilo, and F. M. Spedalieri, Detecting multipartite entanglement, Phys. Rev. A 71, 032333 (2005).

[11] G. Tóth, T. Moroder, and O. Gühne, Evaluating Convex Roof Entanglement Measures, Phys. Rev. Lett. 114, 160501 (2015).

[12] J. Bavaresco, N. Herrera Valencia, C. Klöckl, M. Pivoluska, P. Erker, N. Friis, M. Malik, and M. Huber, Measurements in two bases are sufficient for certifying high-dimensional entanglement, Nat. Phys. 14, 1032 (2018).

[13] X.-M. Hu, W.-B. Xing, B.-H. Liu, Y.-F. Huang, C.-F. Li, G.-C. Guo, P. Erker, and M. Huber, Efficient Generation of High-Dimensional Entanglement through Multipath DownConversion, Phys. Rev. Lett. 125, 090503 (2020).

[14] H.-S. Zhong, Y. Li, W. Li, L.-C. Peng, Z.-E. Su, Y. Hu, Y.-M. He, X. Ding, W. Zhang, H. Li, L. Zhang, Z. Wang, L. You, X.-L. Wang, X. Jiang, L. Li, Y.-A. Chen, N.-L. Liu, C.-Y. Lu, and J.-W. Pan, 12-Photon Entanglement and Scalable Scattershot Boson Sampling with Optimal Entangled-Photon Pairs from Parametric Down-Conversion, Phys. Rev. Lett. 121, 250505 (2018).

[15] M. Malik, M. Erhard, M. Huber, M. Krenn, R. Fickler, and A. Zeilinger, Multi-photon entanglement in high dimensions, Nat. Photonics 10, 248 (2016).

[16] S. T. Flammia and Y.-K. Liu, Direct Fidelity Estimation from Few Pauli Measurements, Phys. Rev. Lett. 106, 230501 (2011).

[17] M. Weilenmann, B. Dive, D. Trillo, E. A. Aguilar, and M. Navascués, Entanglement Detection beyond Measuring Fidelities, Phys. Rev. Lett. 124, 200502 (2020).

[18] M. Weilenmann, E. A. Aguilar, and M. Navascués, Analysis and optimization of quantum adaptive measurement protocols with the framework of preparation games, Nat. Commun. 12, 4553 (2021).
[19] See Supplemental Material at http://link.aps.org/ supplemental/10.1103/PhysRevLett.127.220501 for more theoretical and experimental detail, which includes additional Refs. [20-22].

[20] D. Elkouss and S. Wehner, (Nearly) optimal P values for all Bell inequalities, npj Quantum Inf. 2, 16026 (2016).

[21] M. Araújo, F. Hirsch, and M. T. Quintino, Bell nonlocality with a single shot, Quantum 4, 353 (2020).

[22] N. Johnston, QETLAB: A MATLAB toolbox for quantum entanglement, version 0.9, http://qetlab.com (2016).

[23] MOSEK ApS, The MOSEK optimization toolbox for MATLAB manual. Version 9.2. (2020), https://www.mosek .com.

[24] X.-M. Hu, Y. Guo, B.-H. Liu, Y.-F. Huang, C.-F. Li, and G.-C. Guo, Beating the channel capacity limit for superdense coding with entangled ququarts, Sci. Adv. 4, eaat9304 (2018).

[25] S. Ecker, F. Bouchard, L. Bulla, F. Brandt, O. Kohout, F. Steinlechner, R. Fickler, M. Malik, Y. Guryanova, R. Ursin, and M. Huber, Overcoming Noise in Entanglement Distribution, Phys. Rev. X 9, 041042 (2019).

[26] R. T. Thew, K. Nemoto, A. G. White, and W. J. Munro, Qudit quantum-state tomography, Phys. Rev. A 66, 012303 (2002).

[27] X.-M. Hu, J.-S. Chen, B.-H. Liu, Y. Guo, Y.-F. Huang, Z.-Q. Zhou, Y.-J. Han, C.-F. Li, and G.-C. Guo, Experimental Test of Compatibility-Loophole-Free Contextuality with Spatially Separated Entangled Qutrits, Phys. Rev. Lett. 117, 170403 (2016).

[28] O. Gühne, Y. Mao, and X.-D. Yu, Geometry of Faithful Entanglement, Phys. Rev. Lett. 126, 140503 (2021).

[29] Y. Zhan and H.-K. Lo, Detecting entanglement in unfaithful states, arXiv:2010.06054.

[30] G. Tóth and T. Vértesi, Quantum States with a Positive Partial Transpose are Useful for Metrology, Phys. Rev. Lett. 120, 020506 (2018).

[31] H. C. Nguyen and O. Gühne, Some Quantum Measurements with Three Outcomes Can Reveal Nonclassicality where All Two-Outcome Measurements Fail to Do So, Phys. Rev. Lett. 125, 230402 (2020) 